\title{
Cross-sectional Diameter at End Ventricular Systole
}

National Cancer Institute

\section{Source}

National Cancer Institute. Cross-sectional Diameter at End Ventricular Systole. NCI

Thesaurus. Code C127546.

The cross sectional diameter measured at end ventricular systole. 